\title{
Le traitement judiciaire de la jeunesse délinquante à Genève de 1738 à 1792
}

The Herman Diederiks Prize Essay for 2005 / Lauréat du prix Herman Diederiks 2005

Judicial treatment of young offenders in Geneva from 1738 to 1792

\section{Gianenrico Bernasconi}

\section{(2) OpenEdition} Journals

Édition électronique

URL : https://journals.openedition.org/chs/192

DOI : $10.4000 /$ chs. 192

ISSN : 1663-4837

Éditeur

Librairie Droz

Édition imprimée

Date de publication : 1 juin 2006

Pagination : 5-23

ISBN : 978-2-600-0189-4

ISSN : 1422-0857

Référence électronique

Gianenrico Bernasconi, « Le traitement judiciaire de la jeunesse délinquante à Genève de 1738 à

1792 », Crime, Histoire \& Sociétés / Crime, History \& Societies [En ligne], Vol. 10, n 1 | 2006, mis en ligne le 01 juin 2009, consulté le 25 mars 2022. URL : http://journals.openedition.org/chs/192 ; DOI : https://doi.org/10.4000/chs.192

Ce document a été généré automatiquement le 25 mars 2022

(C) Droz 


\section{Le traitement judiciaire de la jeunesse délinquante à Genève de 1738 à $1792^{1}$}

The Herman Diederiks Prize Essay for 2005 / Lauréat du prix Herman

Diederiks 2005

Judicial treatment of young offenders in Geneva from 1738 to 1792

\section{Gianenrico Bernasconi}

\section{Introduction}

1 Les historiens qui se sont occupés de délinquance juvénile sont arrivés à la conclusion que c'est au cours du XIX ${ }^{e}$ siècle qu'émerge (voire 'est inventée'), la figure sociale et pénale du jeune délinquant ${ }^{2}$. Les réformes du droit pénal, la naissance de la police criminelle, les nouvelles inquiétudes au sujet des 'classes dangereuses' attirent l'attention sur la délinquance juvénile. Tout au long du XIX ${ }^{e}$ siècle, une série d'initiatives politiques, scientifiques et philanthropiques se posent le problème de comprendre et de combattre ce phénomène. Ces projets aboutissent à l'élaboration d'un concept de minorité pénale, à l'instauration de tribunaux des mineurs et de structures carcérales réservées aux jeunes ${ }^{3}$.

Ces transformations mettent justement en lumière l'attention prêtée au cours du XIX ${ }^{\mathrm{e}}$ siècle aux problèmes soulevés par la criminalité juvénile. Toutefois, il est difficile de partager les conclusions de ces études au sujet du traitement de la délinquance juvénile au cours des siècles précédents. Le désintérêt de l'historiographie envers les sources de

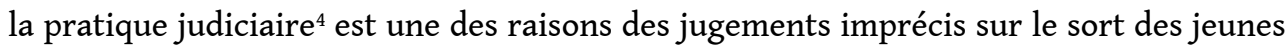
prévenus avant le $\mathrm{XIX}^{\mathrm{e}}$ siècle. Les historiens se sont contentés trop souvent des textes de l'ancienne doctrine criminelle pour évaluer le traitement de l'âge dans le système judiciaire de l'Ancien Régime ${ }^{5}$. 
3 Cette méconnaissance a été par ailleurs soutenue par la diffusion des termes « invention » et « émergence » dans les travaux traitant de l'histoire de la délinquance juvénile. Quoique le syntagme "délinquance juvénile» apparaît dans les sources seulement au XIX ${ }^{\mathrm{e}}$ siècle $^{6}$, l'expression «invention de la délinquance juvénile » doit toutefois faire l'objet d'une discussion. Cela non seulement en raison des débats autour de l'usage du terme d'«invention" dans l'historiographie de l'enfance et de l'adolescence ${ }^{7}$, mais aussi en raison d'une réflexion sur les arguments qui étayent « l'invention de la délinquance juvénile » au cours du XIX ${ }^{\mathrm{e}}$ siècle. H. Shore a récemment observé que les transformations, auxquelles est attribuée l'émergence du problème social et pénal de la délinquance juvénile, s'inscrivent dans une chronologie complexe ${ }^{8}$. La législation réservée aux jeunes prévenus, les institutions spécialisées dans la punition et dans la correction des jeunes, ainsi que l'intervention de l'État dans les familles des « jeunes dépravés » s'enracinent avant le XIX ${ }^{\mathrm{e}}$ siècle $^{9}$.

4 Cet article voudrait contribuer à cette discussion portant sur la chronologie de l'histoire de la délinquance juvénile, en examinant le traitement de l'âge du prévenu dans le système judiciaire genevois de la deuxième moitié du XVIII ${ }^{\mathrm{e}}$ siècle.

5 Les recherches de $M$. Porret à partir des archives judiciaires genevoises ont mis en évidence qu'au cours du XVIII ${ }^{\mathrm{e}}$ siècle la notion de proportionnalité entre crimes et peines, qui avait été l'objet du combat intellectuel mené par les réformateurs du droit des Lumières, s'introduit dans la pratique judiciaire genevoise ${ }^{10}$. Les procureurs généraux qualifient dans leurs réquisitoires les crimes en évaluant les circonstances qui les accompagnent et proposent aux juges des peines « mesurées » à la gravité du crime. L'âge des prévenus figure parmi les circonstances prises en considération par les magistrats.

6 En étudiant les procès instruits à Genève au XVIII ${ }^{e}$ siècle et l'ancienne doctrine criminelle, on peut comprendre comment les magistrats de la deuxième moitié du $\mathrm{XVIII}^{\mathrm{e}}$ siècle affrontent le problème de la jeunesse des prévenus et tiennent compte de la fragilité de l'âge lorsqu'ils proposent des peines aux juges.

\section{L'âge dans la doctrine pénale du XVIII siècle}

7 L'étude du système judiciaire $\mathrm{du} \mathrm{XVIII}^{\mathrm{e}}$ siècle a révélé que parallèlement à la lutte philosophique et politique visant à une réforme du système judiciaire, s'accomplit dans les tribunaux un processus de "protolégalisation » du système pénal, qui va poser des limites à l'arbitraire des juges ${ }^{11}$. Dans la pratique judiciaire se diffuse la nécessité d'établir une proportionnalité entre peines et crimes, qui se réalise à travers une évaluation précise des circonstances. En qualifiant le crime, les circonstances permettent en effet d'en mesurer la gravité et d'attribuer une peine proportionnelle. La "partie publique» doit donc motiver les peines, en les conformant aussi à la jurisprudence, car « il ne suffit pas que la loi commande ce qui est bon et juste en soi », mais il faut encore "qu'elle s'insinue et se grave dans les cœurs par des raisons solides $»^{12}$.

8 À Genève, le procureur général, qui depuis le Règlement de l'illustre médiation (1738), intervient en « grand criminel » pour tout délit puni par une peine capitale, par la mort civile ou encore par toute sanction corporelle ou infamante, joue un rôle central dans le processus de limitation de l'arbitraire. En effet, après avoir vérifié l'authenticité des 
pièces de la procédure, il dresse un réquisitoire, dans lequel il motive la peine qu'il propose aux juges en considérant les circonstances qui qualifient le crime, la jurisprudence genevoise, les lois romaines et la doctrine criminelle. L’âge du prévenu figure parmi les circonstances considérées par les magistrats.

Institué par des Édits, le système judiciaire genevois n'est pas réglé par des codes particuliers. Afin de combler ce vide juridique, les magistrats genevois font référence aux textes législatifs de la monarchie française, notamment à l'Ordonnance criminelle de Saint-Germain-en-Laye (1670) et à l'ancienne tradition doctrinale ${ }^{13}$.

Pour comprendre l'usage, dans la pratique judiciaire, de la circonstance relative à l'âge, il faut d'abord illustrer la doctrine pénale du XVIII ${ }^{\mathrm{e}}$ siècle.

\section{a) Les classes d'âge ${ }^{14}$}

11 S'inspirant du droit romain, l'ancienne doctrine pénale partage les mineurs en trois classes d'âge : les enfants, les impubères et les mineurs de 25 ans ${ }^{15}$.

12 - Les enfants sont les individus qui n'ont pas encore atteint l'âge de 7 ans. Ils sont considérés comme "incapables de malice ». Leur âge ne leur permet pas, en effet, de « considérer le bien et le mal » et, puisqu'il n'y a que « la volonté et la connaissance du mal qu'on fait qui méritent la punition $»^{16}$, ils sont exemptés de tout crime et de toute punition.

13 - Les impubères, les garçons jusqu'à 14 ans et les filles jusqu'à 12 ans, "sont aussi excusables en matière de crime ». Les criminalistes distinguent dans cette tranche d'âge une catégorie particulière, la proximitas pubertatis : «si le crime a été commis dans un âge plus voisin de la puberté que de l'enfance, tel que celui de dix ans pour les filles et de onze ans pour les mâles, alors on peut présumer dans celui qui le commet une capacité suffisante pour discerner le bien et le mal. La loi ne veut pas qu'il soit absolument exempt de peine, mais seulement que cette peine soit moindre que celle qui doit infliger à ceux qui ont commis le crime en pleine puberté ${ }^{17}$.

14 Au sujet de cette distinction, le criminaliste français D. Jousse (1704-1781) propose de considérer le processus de développement des facultés intellectuelles: "pour savoir quand un impubère est proche de l'âge de puberté, cela dépend de la prudence du Juge, qui doit considérer dans ce cas la qualité de la personne, ainsi que la nature et les circonstances du crime $»^{18}$. Même si, en règle générale, les impubères sont considérés comme irresponsables (ou partiellement responsables) par rapport à leurs actes ${ }^{19}$, les pénalistes peuvent appliquer pour certains crimes l'adage juridique malitia supplet aetatem: «il y a des cas où les mineurs peuvent être condamnés en la peine ordinaire... lorsqu'il s'agit d'assassinat à prix d'argent, de vol réitéré, d'usure, et d'autres cas qui proviennent plutôt d'une malice réfléchie que de la jeunesse ou de l'imprudence $»^{20}$. Pour certains crimes particulièrement « atroces", la nature de l'acte dépasse la considération de son intentionnalité. Un acte atroce, comme un crime de lèse-majesté ou un parricide, ne peut, aux yeux des criminalistes, que dévoiler la scélératesse de l'individu qui l'a commis ${ }^{21}$.

15 - Après quatorze ans et jusqu'à 25 ans, «les pubères doivent être punis moins sévèrement que ceux qui sont dans un âge où la raison est parvenue à la pleine maturité, qui est celui de la majorité $»^{22}$. 


\section{b) L'application de la circonstance relative à l'âge : requalification de l'acte ou diminution de la peine}

Ce dispositif juridique nous engage à approfondir l'analyse de la "théorie des circonstances ». Le pénaliste P. F. Muyart de Vouglans (1713-1791) indique deux modalités de traitement de l'âge dans le processus de qualification des crimes et d'établissement des peines :

Il y en a que leur état rend également exempts et de crime et de peine; d'autres, dont l'état, sans diminuer leur crime, peut seulement servir à en faire diminuer la peine ${ }^{23}$.

Cette double fonction de la circonstance relative à l'âge s'inspire de la doctrine théologique selon laquelle «les circonstances peuvent... aggraver (ou diminuer) la malice du péché, en lui laissant sa nature, mais elles peuvent aussi changer sa nature $»^{24}$. Muyart de Vouglans s'occupe de la doctrine relative à l'âge des prévenus en deux parties différentes de son ouvrage Les Loix criminelles en France (1780). D'abord, lorsqu'il traite des «causes qui font cesser le crime ${ }^{25}$, ensuite, lorsqu'il illustre les circonstances " accessoires au fait du crime ", qui, « sans en changer la qualité », servent aux juges pour "augmenter ou diminuer la peine $»^{26}$. L'âge fait "cesser le crime", parce que, comme écrit Jousse, «il n'y a point de crime, sans une volonté de le commettre $\aleph^{27}$. Le discernement du jeune prévenu peut n'être pas suffisamment développé pour que son acte soit considéré comme volontaire et donc comme criminel.

Si le jeune est reconnu comme responsable de ses actes, son âge est tenu en considération comme "circonstance accessoire». Muyart de Vauglans, lorsqu'il traite des «cas où il y a lieu de modérer la peine» invite les juges à tenir compte de la «fougue de la jeunesse » et à atténuer par conséquent la peine ${ }^{28}$.

Dans les réquisitoires des procureurs généraux genevois, on retrouve ce double traitement de l'âge qui peut être à l'origine d'une requalification de l'acte, qui, n'étant pas volontaire, sort de la sphère du crime ou bien d'une atténuation de la peine. En 1773, le procureur général B. Galiffe (1736-1816), appelé à « conclure » au sujet d'un vol domestique commis par B. Ozelet, âgé de 15 ans, tient compte des deux possibilités: "La considération de l'âge semble plutôt s'en tenir à la peine et à une éventuelle réduction, qu'à une autre définition du crime ${ }^{29}$.

20 L'ancienne doctrine pénale développe une double conception de l'âge: l'«âge psychologique ", considéré comme l'état de développement intellectuel et moral du jeune, sur lequel va se prononcer l'arbitraire du juge; l'«âge légal », donnée objective établie par un certificat de baptême, lequel est parfois allégué aux procédures.

21 Le problème de la minorité pénale met en lumière la complexité de l'articulation entre « âge légal » et « âge psychologique » dans la pratique judiciaire.

\section{c) La minorité pénale}

Les criminalistes du XVIII ${ }^{\mathrm{e}}$ siècle fixent, en accord avec le droit romain, le terme de 25 ans pour distinguer minorité et majorité pénale. Ce terme, qui correspond aussi au début de la majorité civile, soulève quelques problèmes. D. Jousse, au moment d'indiquer les classes d'âge qui composent la minorité pénale, fixe l'âge limite à 25 ans. Pourtant, lorsqu'il traite le cas des individus «qui doivent... être punis moins sévèrement que s'ils étaient majeurs, même dans les délits atroces $\aleph^{30}$, il ne considère 
que les mineurs de 20 ans. Cette incohérence dans le texte de Jousse nous oblige à approfondir la problématique inhérente à l'âge qui établit la transition de la minorité à la majorité pénale.

Dans les dictionnaires et dans les encyclopédies du XVII ${ }^{e}$ et du XVIII ${ }^{e}$ siècle, le concept de minorité n'a une signification précise qu'en droit civil. Dans le dictionnaire de A. Furetière (1620-1688), mineur signifie « enfant ou jeune homme qui par les loix, ou les coutumes n'a pas encore l'administration de son bien $»^{31}$. C.-J. de Ferrière (1680-1749), dans son Dictionnaire de droit et pratique $(1734)^{32}$, ne consacre que quelques lignes au problème de la minorité pénale, alors qu'il développe longuement les enjeux de la minorité civile. De même, J.-B. Dénisart (1712-1765), dans la Collection de décisions nouvelles et notions relatives à la jurisprudence (1754-1756) ${ }^{33}$, s'occupe des mineurs principalement au sujet des affaires commerciales et patrimoniales. Dans l'article de l'Encyclopédie, rédigé par A. G. Boucher d'Argis (1708-1780), il apparaît qu'en matière pénale la minorité n'entrâne pas une diminution de la responsabilité :» les mineurs sont aussi traités comme les majeurs, pourvu qu'ils eussent assez de connaissance pour sentir le délit qu'ils commettaient $\aleph^{34}$. Les différences entre un acte criminel et un acte commercial ou patrimonial expliquent le double usage de la minorité face au pénal et au civil : " toute personne qui est proche de l'âge de puberté est capable de dol et par conséquent peut de son chef être accusée et poursuivie en justice. Et il ne faut pas argumenter ici de ce qui s'observe en matière civile, car celui qui est en état de bien ou mal faire, n'a pas toujours assez de prudence pour se conduire et se gouverner dans les affaires civiles $\aleph^{35}$. La majorité en matière criminelle serait donc antérieure à la majorité civile. La puberté marque un tournant dans la responsabilité face aux actes criminels. Aux yeux de Jousse, cette maturité morale ne correspond pas à l'acquisition d'une expérience suffisante pour permettre au pubère de gérer ses " affaires ».

24 L'analyse des conclusions des procureurs généraux genevois confirme l'affirmation de l' » âge psychologique » de la puberté au détriment de celui « légal » de la majorité. En 1773, le procureur général B. Galiffe, dans son réquisitoire contre N. Mariotte, âgé de 20 ans, réaffirme la primauté de l'âge de la puberté sur celle de la majorité: "Relativement à l'âge, quoique les lois statuent que dans la punition des crimes les juges peuvent avoir égard à l'âge des coupables, elles décident cependant que la minorité n'est point une excuse légitime qui doive exempter les coupables de la peine ordinaire des crimes qu'ils ont commis, et cette jurisprudence est celle que Vos Seigneuries ont adoptée dans plusieurs jugements. D’ailleurs le prévenu a passé l'âge de la pleine puberté, il a 20 ans et demi et à cet âge l'on est censé connaître les conséquences des actions et les suites qu'elles peuvent avoir ${ }^{36}$.

À Genève, la transition vers la responsabilité pénale est marquée par une étape fondamentale dans la vie religieuse d'un membre de l'Église réformée : l'admission à la Sainte-Cène. Le catéchisme, qui prépare les jeunes genevois à entrer dans la communauté religieuse, joue un rôle important dans l'éducation morale de la jeunesse $^{37}$. Appelés à qualifier les crimes commis par des jeunes, les magistrats genevois tiennent compte de la formation religieuse pour évaluer leur responsabilité. En 1791, le procureur général J.-F. Butini (1747-1805) observe à propos de J. Calin, âgée de 17 ans:» Elle n'a pas encore été admise en la Sainte Cène et ce n'est qu'après cette admission que les jeunes gens sont censés connaitre la gravité des principaux crimes par lesquels on se rend coupable envers Dieu et envers la Société $»^{38}$. 
L'importance accordée aux déclarations des pasteurs attestant la formation religieuse des jeunes prévenus prouve la volonté des magistrats de limiter l'arbitraire des juges appelés à se prononcer sur leur discernement. Malgré cette tentative de réguler le processus d'évaluation des facultés intellectuelles et morales, la «prudence» des procureurs généraux joue un rôle principal dans l'établissement de la responsabilité du jeune.

\section{3) La qualification judiciaire de la « responsabilité pénale » des jeunes prévenus dans des affaires de "vol domestique »}

28 Les procédures concernant des vols domestiques offrent des sources précieuses pour l'étude du traitement judiciaire de la jeunesse délinquante. La gravité de ces crimes et la sévérité de la peine exigent l'intervention du procureur général, ce qui explique la présence dans ces dossiers criminels des réquisitoires.

29 L'ancienne doctrine pénale répartit le vol selon deux catégories : le vol simple et le vol qualifié. Le premier est celui «qui se fait en cachette et qui n'est accompagné ni d'effraction, ni de port d'armes, ni d'aucune circonstance particulière qui l'aggrave », le second « celui qui est rendu plus grave par les circonstances qui l'accompagnent ${ }^{39}$. Le vol domestique, «qui se fait par un valet, serviteur, ou servante à son maître $»^{40}$, ou encore par des compagnons et des apprentis, appartient à la deuxième catégorie. Il s'agit d'un abus de confiance, circonstance aggravante du vol simple, pour lequel les criminalistes du XVIIIe siècle prescrivent la peine de mort. Le dépouillement des archives judiciaires genevoises a dévoilé une pratique pénale plus nuancée par rapport à la dureté de la doctrine. $\mathrm{M}$. Porret a démontré qu'à Genève « l'usage du gibet recule à partir du milieu du siècle » et que les auteurs d'un vol domestique vont subir désormais une peine infamante, après quoi ils sont bannis à perpétuitét ${ }^{41}$.

Dans leurs réquisitoires, les magistrats évoquent la nécessité de protéger «la fortune des particuliers ", qui dans leurs ateliers d'horlogerie et de bijouterie, doivent se fier à des employés travaillant quotidiennement avec des matières précieuses. Selon le procureur général J.-F. Prévost (1755-1794), « il ne serait que trop facile à des messagers corrompus de faire à leurs maîtres des torts journaliers et d'ouvrir par degrés une grande brèche à leur fortune $\aleph^{42}$. Les magistrats ne se limitent pourtant pas à sauvegarder la richesse des maîtres et à dénoncer les infidélités des apprentis, mais ils se préoccupent aussi du développement de l'activité économique : »Que deviennent ces manufactures importantes si ceux qui doivent un jour les diriger, en faisant l'apprentissage de leur profession, font aussi l'apprentissage du crime? $»^{43}$.

31 Devant conclure sur des délits graves commis par des apprentis ou des messagers, les magistrats évaluent le discernement des jeunes prévenus, en prêtant une attention particulière à la conscience de la valeur du butin et à son usage, aux réponses des jeunes pendant les interrogatoires et à la présence d'un « séducteur ».

\section{a) Le butin}

La valeur du butin est une circonstance importante dans la qualification du vol domestique. Selon Jousse « quoi qu'en général on doive punir de la peine de mort tout 
vol domestique... néanmoins lorsque la chose volée est si modique qu'elle n'est d'aucune considération, il semble qu'on peut diminuer la règle établie $»^{44}$. Les magistrats tiennent compte du butin et évaluent aussi la conscience des jeunes de la valeur des matières précieuses qu'ils ont volées. En 1790, le procureur général J.-F. Prévost constate la modicité du butin volé par B. Ceret, » des boulets de fer, quelques morceaux de laiton et une pièce de dix sols et demi, le tout montant à la valeur d'environ cinq sols ", et observe que " probablement ces infidélités n'eussent pas donné lieu à l'incarcération d'un enfant de 13 à 14 ans $»^{45}$. En 1773, le procureur général $B$. Galiffe propose d'atténuer la peine de J. Ozelet, âgé de 15 ans, parce qu'il « connaissait peu la valeur de l'or qu'il prenait à son maître $»^{46}$. L'auditeur demande à F. Girard, âgé de 11 ans, «s'il ne savait pas que l'or et l'argent sont des matières précieuses, et qu'il faisait un grand tort à son maître en le dérobant $»^{47}$. Le manque d'adresse des jeunes voleurs à cacher les objets volés et l'usage du butin sont d'autres signes de la faiblesse du discernement. En 1771, Galiffe, en motivant une peine contre A. George, voleur âgé de 20 ans, observe chez le prévenu «beaucoup de naïveté, peu d'art et dextérité pour cacher le crime $»^{48}$. De même, l'usage " peu mystérieux » du butin fait par M. Robert, âgé de 14 ans, qui "donne une de ces bagues de laiton à sa sœur... une autre à la servante même de son maitre » et qui vend la troisième "sans avoir dessein d'en faire de l'argent », « n'annonce pas », selon le procureur général J.-R. Tronchin (1710-1792), « qu'il ait cru proprement faire un vol $»^{49}$.

Si la maladresse à cacher le butin et la naïveté dans son usage permettent d'atténuer la malice du jeune délinquant, le cas inverse indique le durcissement criminel de son caractère. G.F. Reymann, âgé de 16 ans, qui n’a que «trop réfléchi sur les moyens de masquer ses vols " ${ }^{50}$, est désigné par le procureur général comme "l'homme dangereux » d'une bande de jeunes voleurs.

La modicité du butin, l'ignorance de la valeur de l'or et l'usage naïf des effets volés atténuent la gravité du crime et diminuent la responsabilité du jeune prévenu. Les magistrats désignent parfois ces infractions avec les termes «infidélité ", «indiscrétion", en abandonnant celui juridique de «vol». Cette transition du vocabulaire illustre une modification de la nature de l'acte, qui se déplace du domaine des crimes punis par la justice à celui des écarts corrigés par la famille : «De telles infidélités à l'âge du prévenu étaient plutôt l'objet de la censure paternelle que l'animadversion des tribunaux $»^{51}$.

\section{b) L'interrogatoire}

Moment central dans le processus d'incrimination, l'interrogatoire a « comme objet de faire preuve de vérité ", à travers l'aveu des circonstances du crime. Les réponses des jeunes prévenus permettent d'évaluer leur développement intellectuel et moral.

Selon Tronchin, «si l'on remarque un degré d'ignorance ou de simplicité qui puisse faire présumer la faiblesse de l'esprit, ce sera une nouvelle raison de mitiger la peine $»^{52}$. Appelé à se prononcer sur les vols commis par B. Rossignol, âgé de 17 ans, il admet qu'il serait « difficile de décider si l'accusé est dans ce cas ». Même s'il a montré dans ses interrogatoires "de la présence d'esprit et une mémoire fidèle", "sa contenance en présence de ses juges n'est pas moins étonnante. Il a montré ni inquiétude, ni dissimulation, ni douleur. Il n'est pas plus tenté d'excuser ses vols que s'ils étaient sans conséquence. Il leur en parle comme il en parlerait à des confidents... 
peut-être, pourrait-on considérer cette espèce d'abandon de lui-même qu'on remarque dans les interrogatoires de l'accusé, comme l'imprudence de cet âge qui ne saurait point encore apprécier les actions, et qui se met point en défense pourvu qu'il ne se croit point en danger ». C'est donc l'« imprudence», ce «manque de précaution, de réflexion et de délibération ", qui convainc le procureur général de prêter égard à l'âge du prévenu. L'«imprudence» de Rossignol est mise au compte de ses facultés intellectuelles: sa "raison naissante", conclut Tronchin, "paraît-même avoir été retardée $»^{53}$.

\section{c) L'« instigateur»}

Dans les ateliers, la promiscuité entre classes d'âge expose les jeunes aux influences néfastes des séducteurs adultes.

Devant se prononcer sur la peine à infliger à $\mathrm{M}$. Chappuis, accusé d'avoir inspiré les vols de J. Malcontent, âgé de 13 ans, Tronchin plaide pour une punition sévère: «que deviendra le caractère de cette partie de la Nation si, par une commisération barbare on ne punissait que faiblement ces hommes lâches qui instruisent ou qui encouragent à l'infidélité une jeunesse déjà trop fragile? $»^{54}$.

Dans les traités pénaux du XVIII ${ }^{e}$ siècle, les responsabilités « de ceux qui conseillent le crime", sont vaguement définies. Selon Muyart de Vouglans «celui qui donne le conseil ne devient punissable, suivant les lois, que dans le cas seulement où il serait prouvé que le conseil a été frauduleux, c'est-à-dire, qu'il a été donné par des vues d'un intérêt personnel ou par haine et vengeance contre celui envers qui le crime aurait été commis.(...) L'on dit qu'il serait prouvé, parce que c'est une règle générale que la fraude ne se présume point et que dans le doute, l'on doit toujours pencher à croire que le conseil a été donné de bonne foi et sans en prévoir les conséquences». Même si le conseil a été qualifié de frauduleux, il faut pourtant démontrer par la suite que le crime a été effectivement la conséquence des incitations $»^{55}$. La gravité du "conseil» ne se mesure pas à l'âge de celui auquel il a été donné. La doctrine ne conçoit donc pas la notion de victime de la "séduction ». L'absence d'une base légale contre l'instigation d'enfants au crime est observé par Tronchin dans ses «Conclusions » contre Chappuis. Le procureur général, après avoir retenu la réitération du vol et son caractère domestique, ajoute un troisième grief » que les Lois n'ont pas prévu et qui, selon moi, l'emporte par sa gravité sur les deux autres; je veux dire la séduction d'un enfant par un homme fait " ${ }^{56}$. La même sévérité ressort des «Conclusions » du procureur général L. Buisson (1705-1787) contre J. Devilette, âgée de 37 ans, accusée d'avoir inspiré le crime commis par A. Grossjean, âgé de 15 ans : « Au crime qu'elle partage avec lui, se joint celui de la séduction d'un jeune homme, dont elle est entièrement cause. Cette séduction considérée soit par rapport à la noirceur qui la caractérise, soit par rapport à ses suites et par conséquent à l'intérêt dont il est pour la société... fait un nouveau crime, que je regarde comme étant beaucoup plus grave que celui de Grossjean $»^{57}$.

La conscience de la valeur des matières précieuses et l'usage du butin, les réponses au cours des interrogatoires, la présence d'un séducteur sont des circonstances qui permettent aux procureurs généraux d'évaluer le discernement des jeunes prévenus et donc leurs responsabilités. Les magistrats, confrontés avec des crimes dans lesquels les jeunes ont été victimes de l'instigation néfaste d'un adulte, dénoncent - comme Tronchin - l'absence d'un dispositif juridique qui protège l'enfance. Il émerge donc 
dans la pratique judiciaire la prise de conscience de la nécessité d'une réforme du système juridique vers une meilleure prise en charge de la faiblesse de l'âge.

41 Cependant, la reconnaissance de la fragilité du discernement n'entraîne que rarement l'annulation de la responsabilité du jeune prévenu et donc la dépénalisation de son crime. Malgré l'atténuation de la gravité du délit, le jeune est condamné. Pour compléter l'analyse du traitement judiciaire de la jeunesse délinquante, il faut alors étudier la pratique pénale.

\section{La pratique pénale}

La peine, proportionnée au crime à travers l'évaluation des circonstances, doit réparer le tort subi par la victime, "contenir les méchants et les engager à éviter le crime " ${ }^{58}$, en restaurant ainsi l'ordre social mis en danger par le délit. À partir de 1760, s'enregistre à Genève une évolution du régime pénal: «la pédagogie de l'effroi abandonne progressivement le châtiment des corps pour investir l'âme des scélérats devenue l'objet nouveau de la pénalité $»^{59}$. Dans les réquisitoires des procureurs généraux genevois, les motivations des peines proposées contre des jeunes prévenus illustrent cette évolution. Si, d'une part, il reste la nécessité d'une peine exemplaire qui sert à endiguer la licence de la jeunesse, d'autre part, se dessinent des nouvelles fonctions de la punition. Les magistrats, conscients de l'impact social de l'infamie, tâche flétrissante de la peine publique qui entraîne la "perte de l'honneur " et l'exclusion sociale, penchent pour une peine qui ne soit pas déshonorante. En 1778, évaluant l'opportunité d'infliger au jeune Aidam, âgé de 17 ans, une peine infamante, le procureur général J.-J. Dunant (1742-1802) observe que « bien loin de le corriger », elle pourrait «flétrir son âme » et « l'éloigner... pour toujours du chemin de la vertu » ${ }^{60}$. J.P. Prevost "conclut» en 1790 une procédure contre L. Guichard, âgé de 18 ans, en affirmant «qu'il ne faut pas infliger une peine flétrissante à un jeune homme qui peut conserver l'espoir de recouvrer un jour ses droits dans l'estime des hommes " ${ }^{61}$. Même si elle ne flétrit pas, la peine doit néanmoins être corrective, toucher le cœur du jeune prévenu et « laisser des fortes impressions au coupable qu'on veut guérir ». En 1766, le procureur général J.-R. Tronchin propose contre M. Bertrand, âgée de 18 ans, une peine "qui frappe fortement son imagination et qui la mette dans l'impuissance de céder à ses penchans ou à ses habitudes ${ }^{62}$.

Dans la motivation des peines, l'âge du prévenu figure parmi les circonstances sur lesquelles les magistrats mesurent la sévérité du châtiment. En 1773, le magistrat chargé du réquisitoire contre Ozelet observe que « ce qui doit nécessairement engager à modérer la peine que le délit qu'il a commis peut mériter, c'est la considération de son âge, puisqu'il n'a pas encore 16 ans $»^{63}$. M. Penard, âgée de 15 ans, est arrêtée, en 1790 , pour des " délits aussi graves ", qui " justifieraient contre elle un jugement très sévère ». Pourtant, le procureur général J.-F. Prévost considère que "l'âge de cet enfant $»$ devrait adoucir la peine ${ }^{64}$.

L'âge auquel font référence les magistrats dans leurs motivations est celui que nous avons défini comme "âge légal». À la différence de l'« âge psychologique », dont la qualification est attribuée à l'arbitraire du juge, l'«âge légal», donnée objective attestée parfois par un acte de baptême, diminue la peine selon un dispositif juridique. En effet, la doctrine, en fixant des classes d'âge sur lesquels le magistrat doit calculer la 
sévérité de la peine, pose les bases pour la régulation du traitement pénal de la jeunesse délinquante.

Dans un système judiciaire dans lequel il n'existe pas de structures punitives réservées aux jeunes délinquants, il faut démontrer comment se réalise cette volonté d'adapter la sévérité de la peine à l'âge du délinquant. La peine du fouet offre à cet égard un exemple intéressant.

\section{a) Le fouet}

Selon l'ancienne doctrine pénale, le fouet est une peine flétrissante qui jette l'infamie sur celui qui la subit. Pourtant, la doctrine prévoit une sorte de fouet qui «quoique corporel, n'emporte point note d'infamie $»^{65}$ : le fouet sous la custode. En effet, ce genre de punition n'est pas administré «par l'exécuteur de la justice, dans les carrefours et places publiques, comme on le pratique à l'égard du fouet ordinaire, mais par le geôlier ou questionnaire, dans la geôle ou chambre de correction, ce qui est moins infamant ».

Dans la pratique pénale genevoise, la sévérité du fouet infligé aux jeunes varie sur une échelle qui en mesure l'infamie selon deux axes indiquant, d'une part, la publicité de la peine et, d'autre part, la qualité du « fustigateur ».

\section{La publicité de la peine}

La tâche flétrissante se fonde sur la publicité de la peine, de sorte que si le prévenu est un jeune dont on espère une possible "régénération", le fouet est pratiqué dans un espace «non-public » : la maison de correction. Eu égard à la proportionnalité entre le crime et la peine, la sévérité du fouet se module sur un axe qui va du plus intérieur et moins infamant au plus extérieur et plus infamant.

En 1750, J. Guay, âgée de 10 ans, prévenue de vol, est condamnée à être fouettée « dans la chambre de la maison de correction $»^{66}$. En 1778, A. Carvé, âgé de 14 ans, poursuivi pour vol, est condamné au fouet "à la maison de correction »" ${ }^{67}$. En 1743, P. Pernette, âgée de 16 ans, prévenue de vol de jardinage, est condamnée au fouet «au bas de l'escalier de la maison de correction $»^{68}$.

50 En revanche, lorsque le crime est grave, le fouet est administré dans un espace public. En 1780, C. Schurmann, âgé de 18 ans, responsable d'un vol qualifié, est condamné à être fouetté "à la place de Molard $"^{69}$. En 1781, J. Gallay, âgé de 16 ans, voleur récidiviste, est condamné à être fouetté « dans tous les carrefours » $»^{70}$.

\section{Le fustigateur}

Dans le traitement pénal genevois de la jeunesse délinquante, on distingue trois catégories de personnes chargées d'administrer le fouet et qui confèrent à ce châtiment une infamie plus ou moins grande. Si être fouetté dans la maison de correction par le geôlier est moins infamant qu'être fouetté par l'exécuteur de la haute justice, le jeune prévenu bénéficie d'une catégorie de "fouetteur» encore moins flétrissante: un membre de sa famille. En 1742, J.-D. Raymond, âgé de 14 ans, accusé d'excès, est condamné à être fustigé à la correction " par quelqu'un de ses parents " ${ }^{71}$. De même $\mathrm{G}$. Alary, âgé de 14 ans, et A. Charles, âgé de 13 ans, prévenus en 1761 de vol, sont condamnés au fouet « par leurs parents $»^{72}$. 


\section{L'intensité de la douleur} l'arbitraire dans le traitement judiciaire de l'âge pendant la deuxième moitié du XVIII ${ }^{e}$ siècle.

\section{a) Jeunes apprentis victimes de leurs maîtres}

Deux cas de règlement infrajudiciaire ${ }^{76}$, pénalisés à la suite de la dénonciation d'une des parties, montrent comment l'exigence de protéger l'âge du prévenu peut passer au deuxième plan par rapport à la volonté de maintenir l'ordre social. Dans ces deux affaires $^{77}$, des jeunes apprentis, chargés de vol domestique, accusent des adultes de recel. Leurs maîtres voudraient résoudre ces contentieux hors des tribunaux, devant des tiers jouant le rôle d'arbitre (les membres de la corporation des orfèvres et un notaire) afin d'éviter l'infamie et les frais de justice. Pourtant les adultes accusés de recel, se sentant calomniés, s'adressent à la justice pour protéger leur honneur. Au cours des procédures, il ressort qu'à l'origine de l'aveu du délit et donc des calomnies, il y a l'extorsion de la confession aux jeunes apprentis de la part de leurs maitres. Interrogé par l'auditeur, Girard, âgé de 11 ans, dénonce les excès de son maître, qui « le

Crime, Histoire \& Sociétés / Crime, History \& Societies, Vol. 10, n | 2006 
prenait par les cheveux et le battit contre la muraille " pour lui faire avouer le vol. La déposition d'une voisine, confirme les déclarations de Girard : « Elle s'est aperçue que le dit Hauser est très violent, qui s'enivre très souvent, qu'elle a souvent entendu qu'il battait son apprenti $»^{78}$. De même, Ceret, âgé de 13 ans, affirme que son maître, en le questionnant, lui «tira un peu les cheveux $»^{79}$. Dans les procédures, la disparition de matières précieuses, dont ont été chargés les jeunes apprentis, reçoit des nouvelles explications. Une voisine déclare que «souvent les ouvriers du Sr Hauser (maître de Girard) sont montés sur le toit du banc qui appartient au mari de la déposante », parce que "c'était depuis les fenêtres du cabinet... que cet or tombait sur le toit du banc ». Quant aux maîtres de Ceret, ils avouent de n'être pas sûrs de ces vols, car leur boutique «avait subi le feu». De ces dépositions, il ressort que la perte d'or serait due à des accidents de travail et pas à un vol. Malgré cette conclusion, les excès contre ces jeunes, à l'origine de l'extorsion d'aveu et des accusations calomnieuses, n'ont pas de suites judiciaires. Les magistrats dans leurs réquisitoires protègent au contraire les maîtres. Le procureur général J.-F. Prévost " conclut » la procédure en proposant de décharger Ceret de l'imputation de vol, "qu'il s'était faite à lui-même et qu'il a rétracté ensuite » (sic!) et de le censurer "grièvement » pour «l'accusation fausse qu'il avait faite contre le Sieur Ducré ». Le magistrat ne tient donc pas compte des responsabilités des maîtres et, au contraire, il se préoccupe de les mettre à l'abri d'éventuelles poursuites: "Je crois important de cacher la sentence dans les termes que je viens d'employer pour éviter toute poursuite en dommages et intérêts contre les Sr. Mare et Veygrassat $»^{80}$.

Les jeunes, victimes des excès et de l'extorsion d'aveu, ne bénéficient d'aucune protection de la part des magistrats, qui veulent éviter les troubles de l'ordre social, qu'entraînerait la condamnation des deux maîtres.

\section{b) Punir les jeunes en fonction de leur origine sociale}

La maison de correction ou discipline, institution d'enfermement fondée à Genève en 1631, devient, au cours du XVIII ${ }^{\mathrm{e}}$ siècle, une structure dans laquelle les jeunes prévenus sont enfermés pour des longues périodes de détention à la suite d'un arrêt judiciaire ${ }^{81}$.

61 En 1790, M. Penard, tout en ayant démontré, selon le procureur général J.-F. Prévost, un "penchant au crime invétéré », est condamnée à être enfermée dans la maison de correction, en considération de son âge et « sa qualité de Genevoise » ${ }^{82}$. En revanche, M. Bertand, âgée de 18 ans, arrêtée pour vol en 1766, pour laquelle le procureur général envisage dans un premier temps l'enfermement dans la même institution, est fouettée au Molard et bannie, car elle est catholique et étrangère ${ }^{83}$. Le même sort avait frappé l'année précédente, B. Rossignol qui «malheureusement est catholique romain ${ }^{84}$. Ces deux cas démontrent que l'enfermement dans la maison de correction est l'alternative réservée aux jeunes genevois de confession réformée, en lieu et place de peines infamantes et douloureuses. L'âge joue ici un rôle secondaire par rapport à l'appartenance du jeune au corps citadin, circonstance qui détermine, dans ces cas, le choix de la peine et révèle une différence de traitement judiciaire entre jeunes genevois et jeunes étrangers.

62 La volonté des magistrats de protéger l'ordre social au détriment de l'âge de la victime ou encore l'influence de l'appartenance sociale dans le choix des peines destinées à des jeunes prévenus mettent en évidence les limites du traitement de l'âge dans un système 
judiciaire réglé par la "théorie des circonstances». Dans la comptabilité des circonstances aggravantes et atténuantes dressée par les magistrats afin de limiter l'arbitraire, l'âge figure comme une des circonstances qui qualifient le crime et déterminent la peine. Cette « logique d'incrimination » est à l'origine d'une « impasse conceptuelle $»^{85}$, car si d'une part elle établit une proportionnalité entre crime et peine, elle aboutit d'autre part, à travers un recensement des circonstances pour chaque crime, à une individualisation de la peine qui empêche l'émergence de principes juridiques universels. C'est dans cette perspective qu'il faut comprendre les limites juridiques posées à la définition d'un principe légal de traitement de la jeunesse.

\section{Conclusions}

Inspirée par l'héritage légal romain et par la tradition casuistique, l'ancienne doctrine criminelle prévoit une double fonction de l'âge qui peut être une « cause qui fait cesser le crime » ou une « circonstance accessoire au fait du crime ». Dans le premier cas, l'âge reflète le développement du discernement du jeune prévenu, en fonction duquel son acte peut être retenu comme volontaire et donc comme criminel. Dans le deuxième, l'âge est traité comme une raison pour atténuer la peine selon le classement prescrit par la doctrine. Nous avons défini le premier comme «âge psychologique », qualifié par l'arbitraire du magistrat, le deuxième comme "âge légal », donnée objective attestée souvent par un acte de baptême.

De l'étude des procédures criminelles émerge l'attention avec laquelle les magistrats évaluent les circonstances qui qualifient le discernement du jeune prévenu (conscience de la valeur du butin et son usage, réponses au cours des interrogatoires, présence d'un séducteur). L'importance accordée aux attestations des pasteurs sur l'instruction religieuse et morale des jeunes prouve cette volonté de régler la qualification de leur " âge psychologique », en posant ainsi des limites à l'arbitraire des juges.

Même si le discernement constitue un principe fondamental dans le traitement judiciaire des jeunes, nous avons constaté que rarement la faiblesse des facultés intellectuelles et morales est à l'origine d'une dépénalisation des crimes. Les magistrats tiennent compte par contre de l'«âge légal», afin de proposer une peine "proportionnelle» à la jeunesse du prévenu, dont les motivations rappellent constamment la nécessité de favoriser la "régénérescence» morale et sociale des jeunes « déréglés ». L'analyse de la peine du fouet montre l'existence d'une technologie punitive qui permet d'adapter la sévérité du châtiment à l'âge du délinquant.

Dans l'ancienne pratique judiciaire, malgré la reconnaissance de l'âge comme circonstance atténuante, il reste pourtant une appréhension sociale du crime, mise en évidence par le traitement de l'extorsion d'aveu au détriment des jeunes apprentis et par une régime pénal différent en fonction de l'origine sociale du jeune prévenu.

67 En 1791, avec la promulgation du code pénal, un pas fondamental est accompli vers l'élaboration du principe légal de la minorité. La lecture du texte de 1791 met néanmoins en évidence les continuités avec le dispositif juridique appliqué dans le traitement judiciaire de la jeunesse délinquante au cours du XVIII ${ }^{e}$ siècle. Dans les articles 1-4 du Titre V (De l'influence de l'âge des condamnés sur la nature et la durée des peines) du code de 1791, qui traitent du problème du discernement et de l'influence de la peine sur l'âge du prévenu, on retrouve les principes d'« âge psychologique » et 
d'«âge légal» qui marquent la doctrine et la pratique judiciaire de la fin de l'Ancien Régime.

Cette continuité et l'attention prêtée à l'âge des prévenus par les magistrats genevois nous invitent à inscrire l'étude du traitement judiciaire de la jeunesse délinquante dans une chronologie qui dépasse la césure du XIX ${ }^{\mathrm{e}}$ siècle généralement adoptée et à élargir l'investigation d'autres pratiques correctionnelles concernant l'enfance.

\section{BIBLIOGRAPHIE}

\section{A. Sources imprimées}

Code pénal (1791) et Code pénal (1810), in Lascoumes, P., Poncela, P., Lenoël, P., Au nom de l'ordre : une histoire politique du code pénal, Paris, Hachette, 1989.

Dénisart, J.-B., Collection de décisions nouvelles et notions relatives à la jurisprudence, Paris, 1754-1756. Dictionnaire universel françois et latin, vulgairement appelé Dictionnaire de Trevaux, Nancy, 1690.

Encyclopédie, ou Dictionnaire raisonné des sciences, des arts et des métiers, par une société des genres de lettres..., Paris-Neuchâtel, 1751-1772.

Ferrière, C.-J. de, Dictionnaire de droit et de pratique..., Toulouse, 1779.

Furetière, A., Dictionnaire universel..., La Haye, 1690.

Jousse, D., Traité de la justice criminelle de France, Paris, 1771.

Muyart de Vouglans, P.-F., Institutes au Droit criminel ou Principes généraux sur ces matières, suivant le droit civil, canonique, et la jurisprudence du Royaume, avec un Traité particulier des crimes, Paris, 1757.

Muyart de Vouglans, P.-F., Les lois criminelles de France dans leur ordre naturel, Paris, 1780 et Neuchâtel, 1781.

\section{B. Travaux}

Ariès, P., L'enfant et la vie familiale sous l'Ancien Régime, Paris, Seuil, 1972, (1960).

Bach, P., La délinquance juvénile à Genève de 1740 à 1750 et de 1786 à 1798, Genève, Université de Genève, Faculté de Lettres, 1974 (Mémoire de licence dactylographié).

Barras-Dorsaz, A.-M., Un mode de répression aux $\mathrm{XVII}^{\mathrm{e}}$ et $\mathrm{XVIII}^{\mathrm{e}}$ siècles : la maison de discipline, in Lescaze, B. (éd.), Sauver l'âme, nourrir le corps : de l'Hôpital général à l'Hospice général de Genève, 1535-1985, Genève, Hospice Général, 1985,pp. 72-112.

Beattie, J. M., Crime and the Courts in England, 1660-1800, Princeton (N. J.), Princeton University Press, 1986. 
Bongert, Y., Délinquance juvénile et responsabilité pénale du mineur au XVIII ${ }^{\mathrm{e}}$ siècle, in Abbiateci, A. (éd.), Crimes et criminalité en France XVII ${ }^{e}$-XVIII ${ }^{e}$ siècles, Cahiers des Annales, Paris, A. Colin, 1971, 33, pp. 49-90.

Briegel, F., La clémence du glaive : plaidoyer pour les criminels au siècle des Lumières à Genève, Crime, Histoire \& Sociétés, 2000, 4, 1, pp. 9-29.

Cunningham, H., Histories of Childhood, American Historical Review, 1998, October, pp. 1195-1208.

Dekker, J., The Will to Change the Child: Re-education Homes for Children at Risk in Nineteenth Century Western Europe, Frankfurt am Main, Bern, P. Lang, 2001.

Dupont-Bouchat, M.-S., Pierre, É. (dir.), Enfance et justice au XIXe siècle : essais d'histoire comparée de la protection de l'enfance, 1820-1914: France, Belgique, Pays-Bas, Canada, Paris, Presses universitaires de France, 2001.

Fecteau, J.-M., Ménard, S., Trépanier, J., Strimelle, V., Une politique de l'enfance délinquante et en danger : la mise en place des écoles de réforme et d'industrie au Québec (1840-1873), Crime, Histoire \& Sociétés, 1998, 2, 1, pp. 75-110.

Foucault, M., Surveiller et punir. Naissance de la prison, Paris, Gallimard, 1975.

Garnot, B., (dir.), L'infrajudiciaire du Moyen-Âge à l'époque contemporaine. Actes du colloque de Dijon, 5 \& 6 octobre 1995, Dijon, Éditions universitaires de Dijon, 1996. Histoire de la justice, 1999, 11, pp. 225-243.

Garnot, B., Justice, infrajustice, parajustice et extrajustice dans la France d'Ancien Régime, Crime, Histoire \& Sociétés, 2000, 4, 1, pp. 103-120.

Griffiths, P., Juvenile Delinquency in Time, in Cox P., Shore, H. (Eds), Becoming Delinquent : British and European Youth, 1650-1950, Aldershot, Ashgate Dartmouth, 2002, pp. 22-40.

Henriot, J., Note sur la date et le sens de l'apparition du mot responsabilité, Archives de philosophie du droit : La responsabilité, 1977, 22, pp. 59-62.

King, P., Noël, J., Les origines du 'Problème de la délinquance juvénile' : la multiplication des poursuites contre des mineurs à Londres à la fin du XVIII ${ }^{\mathrm{e}}$ siècle et au début du XIX ${ }^{\mathrm{e}}$ siècle, Déviance et Société, 1994, 18, pp. 3-29.

King, P., The Rise of Juvenile Delinquency in England 1780-1840 : Changing Patterns of Perception and Prosecution, Past and Present, 1998, 158, pp. 116-166.

Krausman, B.-A., Adolescence as a Cultural Invention : Philippe Ariès and the Sociology of Youth, History of the Human Sciences, 1995, 8, 2, pp. 69-89.

Laingui, A., La responsabilité pénale dans l'ancien droit (XVI $-\mathrm{XVIII}^{e}$ siècles), Paris, Librairie générale de droit et jurisprudence, 1970.

Laingui, A., L'homme criminel dans l'Ancien Droit, Revue de science criminelle et de droit pénal comparé, 1983, 1, pp. 15-35.

Magarey, S., The Invention of Juvenile Delinquency in Early Nineteenth-Century England, Labour History, 1978, 34, pp. 325-345.

May, M., A Child Punishment for a Child's Crime : The Reformatory and Industrial School Movement in Britain, 1780-1860, London, University of London, 1981 (PhD Thesis).

Montandon, I., La société des catéchumènes ou société pour faciliter l'instruction de la jeunesse (1736-1850) à Genève pendant le XVIII ${ }^{e}$ siècle, Genève, Université de Genève, 1981 (mémoire de licence dactylographié). 
Nilan, C., Hapless Innocence and Precious Perversity : Construction of the Child Criminal in Late Eighteenth and Early Nineteenth-Century France, Proceedings of the Annual Meeting of the Western Society for French History, 1997, 24, pp. 81-91.

Perrenoud, A., Les réalités humaines, in Piuz, A.-M., Mottu-Weber, L. (Eds), L'économie genevoise de la Réforme à la fin de l'Ancien Régime (XVI-XVIII siècles), Genève, Georg, Société d'histoire et d'archéologie de Genève, 1990, pp. 43-82.

Perrin, B., La minorité pénale en droit romain et dans les législations européennes antérieures, in Donnedieu de Vabres, H., Ancel, M. (Eds), Le problème de l'enfance délinquante. L'enfant devant la loi et la justice pénale, Paris, Librairie du Recueil Siray, 1947, pp. 11-94.

Perrot, M., Quand la société a peur de sa jeunesse au XIX ${ }^{\mathrm{e}}$ siècle, in Proust, F. (éd.), Les jeunes et les autres, Vaucresson, CRIV, 1986, pp. 19-28.

Perrot, M., Adolescence, un pluriel à l'étude des historiens, Adolescence, 1985, 3, 1, pp. 42-72.

Porret, M., Les circonstances aggravantes du vol domestique dans la société de l'Ancien Régime, selon les réquisitoires des procureurs généraux de Genève (XVIII ${ }^{\mathrm{e}}$ siècle), in Garnot, B. (dir.), Ordre moral et délinquance de l'antiquité au XX $X^{e}$ siècle. Actes du colloque de Dijon (7-8 octobre 1993), Dijon, Éditions universitaires de Dijon, 1994a, pp. 295-302.

Porret, M., Effrayer le crime par la terreur des châtiments : la pédagogie de l'effroi chez quelques criminalistes du XVIII ${ }^{e}$ siècle, in Bechtold, J., Porret, M. (Eds), La peur au XVIII ${ }^{e}$ siècle : discours, représentations, pratiques, Genève, Droz, 1994b, pp. 277-285.

Porret, M., Le crime et ses circonstances : de l'esprit de l'arbitraire au Siècle des Lumières selon les réquisitoires des procureurs généraux de Genève, Genève, Droz, 1995.

Porret, M., Une peine en juste proportion du crime : le rôle du procureur général de Genève dans la limitation de l'arbitraire (1760-1790), in Porret, M. (éd.), Beccaria et la culture juridique des Lumières, Genève, 1997, pp. 253-276.

Rawling, P., Crime and Power, A History of Criminal Justice, 1688-1998, London, Longman, 1999.

Ruchat, M., L'oiseau et le cachot : naissance de l'éducation correctionnelle en Suisse romande, 1800-1913, Genève, Zoé, 1993.

Ruchat, M., Pédagogie de la conscience. De l'école des pauvres de Hofwil à la colonie agricole de Serix-sur-Oron en Suisse protestante, Sociétés et représentations : les cahiers du CREDHESS, 1996, 3, pp. 269-276.

Schnapper, B., Les peines arbitraires du XIII ${ }^{\mathrm{e}}$ au XVIII ${ }^{\mathrm{e}}$ siècle (doctrines savantes et usages français), Revue d'histoire du droit, 1973, 41, pp. 237-277; 1974, 42, pp. 81-112.

Shore, H., Artful Dodgers, Youth and Crime in Early Nineteenth-century London, Woodbridge, The Boydell Press, 1999.

Shore, H., Cox, P., Re-inventing the Juvenile Delinquent in Britain and Europe 1650-1950, in Cox P., Shore H. (Eds), Becoming Delinquent : British and European Youth, 1650-1950, Aldershot, Ashgate Dartmouth, 2002, pp. 1-22.

Thiercé, A., Histoire de l'adolescence (1850-1914), Paris, Belin, 1999. 


\section{NOTES}

1. Cet article a été tiré du mémoire de licence, «Entre indulgence et répression : Filous, vauriens et 'faiseurs de gattes'. Jeunes délinquants devant la justice genevoise 1738-1792 », Genève, Faculté des Lettres, 2000. Je remercie Michel Porret, directeur de cette recherche, Marzio Bernasconi, Marco Cicchini et Samuel Dubosson pour la lecture attentive et critique de ce texte.

2. Magarey (1978); May (1981); Ruchat (1993); King, Noël (1994); King (1998); Shore (1999).

3. À ce sujet v. Ruchat (1993); King (1998); Fecteau, Ménard, Trépanier, Strimelle (1998); Shore (1999); Thiercé (1999); Dupont-Bouchat, Pierre (dir.) (2001).

4. V. les critiques formulées par King et Noël (1994).

5. V. par exemple Rawling (1999, p. 96).

6. Nos recherches dans les archives judiciaires genevoises confirment cette chronologie.

7. V. Cunningham (1998); Griffiths (2002, p. 24); Krausman (1995) à propos de l'ouvrage d'Ariès, L'enfance et la vie familiale sous l'Ancien Régime (1960).

8. Shore (2002, p. 6).

9. Shore (2002, p. 7 et ss.).

10. Porret (1995).

11. Au sujet du processus de " protolégalisation » du système pénal v. Porret $(1995,1997)$. Pour une analyse de l'histoire juridique du système arbitraire v. Schnapper $(1973,1974)$.

12. Jousse (1771, I, viii).

13. Porret (1995, pp. 52-53).

14. Au sujet de l'histoire de la minorité pénale, v. Perrin (1947) et Laingui (1970).

15. Jousse (1771, II, p. 615).

16. Muyart de Vouglans (1757, p. 74).

17. Muyart de Vouglans (1781, I, p. 24).

18. Jousse (1771, II, p. 617).

19. Le mot "responsabilité », qui constitue le concept juridique central dans l'évaluation du discernement des jeunes délinquants, n'apparaît dans le vocabulaire juridique français qu'à la fin du XVIII ${ }^{\mathrm{e}}$ siècle. L'absence d'un outillage terminologique relatif à la responsabilité pénale accroît l'intérêt pour l'étude des réquisitoires des procureurs généraux genevois sur l'âge traité comme circonstance atténuante. Au sujet du mot « responsabilité », v. Henriot (1977, pp. 59-62).

20. Jousse (1771, II, p. 618).

21. Laingui (1970, p. 239).

22. Muyart de Vouglans (1781, I, p. 24).

23. Muyart de Vouglans (1781, I, p. 19).

24. Laingui (1983, p. 33).

25. Muyart de Vouglans (1780, pp. 26-27).

26. Muyart de Vouglans (1780, p. 19 et p. 47).

27. Jousse (1771, I, p. 10).

28. Muyart de Vouglans (1780, p. 42).

29. PC 12467, 1773, vol et recel d'or, «Conclusions ».Tous les procès criminels cités dans cet article sont conservés aux Archives d'État de Genève (AEG) et classés dans la série 1. Nous nous contenterons d'abréger les références en ne donnant que le numéro de la procédure ou de l'information (PC), l'année de son ouverture, ainsi que le chef d'accusation figurant sur le « Registre des procès et informations instruits entre 1396 et 1817 » 5 vol.). Toutes nos sources manuscrites ont été retranscrites selon les règles de l'orthographe contemporaine.

30. Jousse (1771, II, p. 617). À ce sujet v. Bongert (1971, p. 72).

31. Furetière (1690, II, art. « mineurs »).

32. Ferrière (1779, II, art. « mineurs »).

33. Dénisart (1754-1756, II, art. « mineurs »). 
34. Encyclopédie ou Dictionnaire raisonné (1766, X, art. « mineur »).

35. Jousse (1771, III, p. 47).

36. PC 12507, 1773, coup de rasoir à un particulier, «Conclusions ».

37. Au sujet de la création de la Société des catéchumènes au XVIII ${ }^{\mathrm{e}}$ siècle, v. Montandon (1981).

38. PC 16375, 1791, vol d'un paire de boucles d'argent, « Conclusions ».

39. Jousse (1771, IV, p. 202).

40. Jousse (1771, IV, p. 202).

41. Porret (1994a, p. 299).

42. PC 16273, 1790, vol, « Conclusions ».

43. PC 10725, 1759, vol et complicité de vol, « Conclusions ».

44. Jousse (1771, IV, p. 204).

45. PC 16064, 1790, vol d'or chez son maître, «Conclusions ».

46. PC 12467, 1773, vol et recel d'or, « Conclusions».

47. PC 10974, 1760, vol.

48. PC 12112, 1771, vol ou soupçon de vol, «Conclusions ».

49. PC 10725, 1760, vol et complicité de vol, «Conclusions ».

50. PC 16275, 1790, vol, «Conclusions ».

51. PC 16064, 1790, vol d'or chez son maître, «Conclusions ».

52. PC 11438, 1765, vol, « Conclusions».

53. PC 11438, 1765, vol, « Conclusions ».

54. PC 10725, 1759, vol et complicité de vol, «Conclusions ».

55. Muyart de Vouglans (1781, I, p. 7).

56. PC 10725, 1759, vol et complicité de vol, «Conclusions ».

57. PC 10005, 1753, vol et recel, « Conclusions».

58. Jousse (1771, IV, p. II).

59. Porret (1994b, p. 60).

60. PC 13182, 1778, vol nocturne de montre, «Conclusions ».

61. PC 16273, 1790, vol, « Conclusions ».

62. PC 11522, 1766, vol, « Conclusions ».

63. PC 12467, 1773, vol et recel d'or, "Conclusions »,

64. PC 15954, 1790, vol et recel, «Conclusions».

65. Jousse (1771, I, p. 58).

66. PC 9694,1750 , vol.

67. PC 13107, 1778, vol.

68. PC 8977, 1743, vol de jardinage.

69. PC 13558, 1780, vol nocturne avec effraction.

70. PC 13678, 1781, vols divers.

71. PC 8841, 1742, excès.

72. PC 10878, 1761, vols.

73. PC 16275, 1790, vol, « Conclusions ».

74. PC 8728, 1740, vol.

75. PC 8833, 1741, larcin.

76. Au sujet de l'infrajudiciaire, v. Garnot (1996, 1999, pp. 234-238, 2000, pp. 105-107).

77. PC 10974, 1760, vol, «Conclusions » et PC 16064, 1790, vol d'or chez son maître.

78. PC 10974, 1760, vol.

79. PC 16064, 1790, vol d'or chez son maître.

80. PC 16064, 1790, vol d'or chez son maître, «Conclusions ».

81. Barras-Dorsaz (1985, p. 103).

82. PC 15914, 1790, vol et recel, « Conclusions».

83. PC 11522, 1766, vol et recel, « Conclusions ». 
84. PC 11438, 1765, vol, «Conclusions ».

85. Porret (1995, p. 436).

\section{RÉSUMÉS}

$\mathrm{Au}$ cours des dernières années, la nécessité de dépasser la frontière chronologique qui situe la «naissance» de la délinquance juvénile au XIX ${ }^{\mathrm{e}}$ siècle s'est affirmée dans le débat historiographique. Certes le dispositif légal et institutionnel réservé aux mineurs se met en place au XIX ${ }^{\mathrm{e}}$ siècle, cependant l'étude de l'ancienne doctrine et des procédures criminelles genevoises de 1738 à 1792 révèle une pratique judiciaire sensible aux problèmes posés par la criminalité juvénile. Les magistrats genevois, héritiers d'une culture juridique marquée par le jus romanum, tiennent compte de l'âge du prévenu, soit comme "cause qui fait cesser le crime », soit comme "raison pour diminuer la gravité de la peine». Dans les procédures émergent donc deux concepts d'âge : "psychologique », qui rend compte du discernement du prévenu, «légal », qui indique son âge biologique. La pratique pénale dévoile aussi une prise de conscience de la nécessité de protéger la "fragilité de l'âge », en distinguant ainsi les jeunes délinquants des prévenus adultes. Cette modernité se heurte cependant à un système judiciaire dans lequel l'arbitraire des juges et le rang social des prévenus continuent à influencer le choix et la motivation des peines.

In recent years the historiographical debate on juvenile delinquency showed the necessity to transcend the chronological barrier limiting the study of this phenomenon to the period beginning with the birth of a system of legal offenses and punishments. Even though it is only in the $\mathrm{XIX}^{\text {th }}$ century that a legal and institutional regime dedicated to minors was born, the study of legal doctrine and criminal procedures in Geneva from 1738 to 1792 shows that judicial practice is sensitive to the youth of the offender.

Genevan magistrates, heirs of a judicial culture influenced by the jus romanum, take into account the age of the accused, either as a "cause which revokes the crime" or as a "ground for diminishing the gravity of the crime». Two different concepts become manifest: "psychological» age, affecting the ability to discern right from wrong, and the biological, «legal» age. Penal practice also shows a realization of the need to protect «the fragility of youth» by distinguishing between young and adult offenders. However, this modernization conflicts with a judicial system where arbitrary rulings and the social ranking of the accused continue to influence the choice and severity of punishments.

\section{AUTEUR}

\section{GIANENRICO BERNASCONI}

Wülischstraße 26, (Hinterhof), D-10245 Berlin, bernasconich73@hotmail.com Gianenrico Bernasconi a étudié l'histoire moderne à Genève. Il prépare une thèse intitulée « L'objet portatif : production, consommation et représentations dans l'espace européen préindustriel », en co-tutelle entre l'Université Paris 1 (Panthéon-Sorbonne) et le Hermann von 
Helmholtz-Zentrum für Kulturtechnik de l'Humboldt Universität de Berlin. Il a été boursier au Hermann von Helmholtz-Zentrum für Kulturtechnik (Humboldt Universität de Berlin). 